\title{
A New Model for Understanding Mechanisms of Biological Signaling: Direct Transport via Cytonemes
}

\author{
Hamid Teimouri and Anatoly B. Kolomeisky* \\ Department of Chemistry and Center for Theoretical Biological Physics, Rice University, \\ Houston, TX 77005-1892 \\ E-mail: tolya@rice.edu
}

\begin{abstract}
Biological signaling is a crucial natural process that governs the formation of all multicellular organisms. It relies on efficient and fast transfer of information between different cells and tissues. It has been presumed for a long time that these long-distance communications in most systems can take place only indirectly via the diffusion of signaling molecules, also known as morphogens, through the extracellular fluid. However, recent experiments indicate that there is also an alternative direct delivery mechanism. It utilizes dynamic tubular cellular extensions, called cytonemes, that directly connect cells, supporting the flux of morphogens to specific locations. We present a first quantitative analysis of the cytoneme-mediated mechanism of biological signaling. Dynamics of the formation of signaling molecule profiles, which are also known as morphogen gradients, is discussed. It is found that the direct-delivery mechanism is more robust with respect to fluctuations in comparison with the passive diffusion mechanism. In addition, we show that the direct transport of morphogens through cytonemes
\end{abstract}

\footnotetext{
*To whom correspondence should be addressed
} 
simultaneously delivers the information to all cells, which is also different from the diffusional indirect delivery. However, it requires energy dissipation and it might be less efficient at large distances due to intermolecular interactions of signaling molecules.

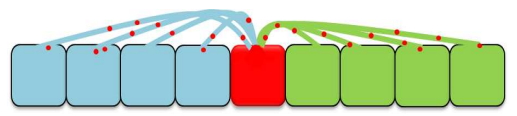

Figure 0: TOC Graphic 
The formation of multi-cellular organisms is one of the most fundamental and mysterious phenomena in nature. ${ }^{1,2}$ In this process, a small number of genetically identical embryo cells via cascades of biochemical and biophysical transitions are transformed into a fully developed organism with a complex structure of tissues and organs. It is now widely accepted that the central role in the biological development, that leads to a complex spatio-temporal patterning in living systems, is played by multiple signaling molecules or morphogens. ${ }^{1-5}$ These biological signaling molecules develop non-uniform concentration profiles, known as morphogen gradients. Embryo cells interact with morphogens, and different genes are activated or suppressed depending on the local concentration of signaling molecules. This leads to the morphological and functional differentiation of tissues and organs. ${ }^{1,2,4}$ A large number of experimental and theoretical investigations on the formation and functioning of morphogen gradients has appeared in recent years. ${ }^{4-19,21,22}$ However, many aspects of biological signaling remain not well understood. ${ }^{4,6}$

One of the most important problems of the biological development is to understand the mechanisms of the morphogen gradients formation. A dominating view in the field is that the profiles of signaling molecules are created by several reaction-diffusion processes. ${ }^{4-6}$ The simplest and most popular idea is known as a synthesis-diffusion-degradation (SDD) mechanism. ${ }^{5,6,9}$ It argues that the formation of signaling molecules profiles starts with a local production at the specific regions of the embryo, then morphogens diffuse in the extracellular fluid along the embryo cells, and finally some of them get captured by receptors on these cells and removed from the system via degradation. The SDD mechanism predicts the exponentially decaying signaling profiles, which qualitatively agrees with many experimental observations. ${ }^{4,5,8-10}$

Despite common use of the SDD model and related approaches for many biological systems, there is a growing number of experimental observations, suggesting that the indirect delivery of the morphogens via diffusion might not be the only mechanism in the transportation of morphogenetic signals. ${ }^{6,22,23,34}$ It has been argued that the complex environment of the embryo systems might prevent the passive free diffusion from establishing distinguishable morphogen gradients at different regions. ${ }^{6,22}$ An alternative direct delivery mechanism of signaling molecules that employs 
cytonemes has been proposed. ${ }^{6,21,24,29}$ Cytonemes are dynamic cellular extensions with the length varying from 1 to $100 \mu \mathrm{m}$ and with the diameter of $\sim 100 \mathrm{~nm} .^{22}$ They have been observed in multiple biological systems but in many cases their functions are not clear. ${ }^{22,23,25,26,28,30,33}$ These tubular objects are supported by actin networks, they can extend and retract quite fast, and their tips can attach to other cells. ${ }^{22}$ The idea behind the direct delivery mechanism is that the morphogens are moving along the cytonemes, probably along the actin filaments with the help from motor proteins, starting from the source cell directly to the specific cell. ${ }^{6,26}$ It is schematically shown in Fig. 1. The advantage of using cytonemes to deliver the morphogenetic signaling molecules is that they can easily adapt to a complex topography of embryo systems, providing a much more precise transfer of information to underlying cells. ${ }^{6}$

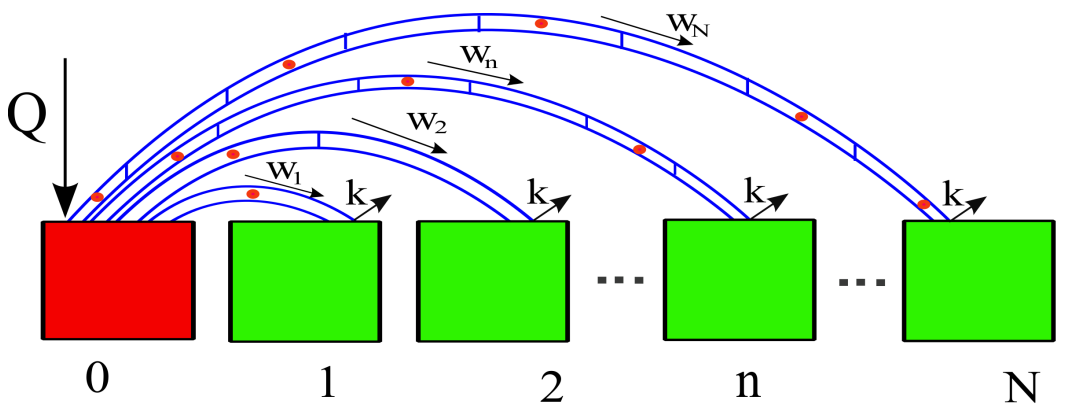

Figure 1: Schematic picture for the direct delivery of morphogens. Squares labeled as $n=0,1, \ldots N$ correspond to embryo cells. The red square $(n=0)$ is a source cell, while green squares $(n>0)$ are target cells. Signaling molecules, shown as small red circles, move along tube that starts in the source cell and ends at the target cells $n$ with the rate $w_{n}$. Morphogens are produced with the rate $Q$ at the source cell. At the target cells they are degraded with the rate $k$.

While various theoretical models have been proposed to describe the formation of the morphogen gradients via free extracellular diffusion, ${ }^{5,16-19}$ there is no detailed theoretical framework for analyzing the direct delivery mechanism (although one should mention recent quantitative studies presented in Ref. ${ }^{20}$ ). In this paper, we address this problem by developing a simple physicalchemical quantitative approach to explain the direct transportation of morphogenetic signals. Our discrete-state stochastic model takes into account the most relevant chemical and physical processes in the system, and it predicts several important dynamic features that can be tested experimentally. Our calculations show that the morphogen gradients created by the direct delivery 
mechanism strongly depend on transportation rates along the cytonemes. We also find a surprising result that the stationary density profiles at all embryo cells are established at the same time, in contrast with the free extracellular diffusion route. This leads to an important conclusion that the direct delivery via cytonemes is more robust mechanism for the formation of signaling molecules profiles. But our analysis also suggests that the direct delivery route might be energetically less efficient, and its efficiency might be also lowered by intermolecular interactions.

Our theoretical model is presented in Fig. 1. It is assumed that there are $N+1$ embryo cells in the system (squares in Fig. 1). One of them (red square, $n=0$ ) is a source cell where morphogens are produced with a rate $Q$. The source cell also generates $N$ cytonemes that extend and attach to each of the target cell (green squares in Fig. 1). The number of cytoneme contact between cells can vary, but for simplicity we can assume that the source cell has only one connection to each target cell - see Fig. 1. Morphogen molecules (small red circles in Fig. 1) are transported to the $n$-th cell with a rate $w_{n}(n=1,2, \ldots, N)$. When the signaling molecules reach their target cells they are degraded with a rate $k$. This is the simplest scheme that takes into account most relevant processes in the systems such as the production of morphogens in the specific cells, the transportation along the cytonemes to specific target cells and the removal of signaling molecules at the targets.

To compute the dynamic properties of the direct delivery mechanism, it is convenient to adapt a single-molecule view here. We define then $P_{n}(t)$ as a probability of finding a signaling molecule at the cell $n$ at time $t$. This can be viewed as proportional to a density or concentration of morphogens at given cell. The temporal evolution of this quantity is governed by a set of master equations:

$$
\frac{d P_{0}(t)}{d t}=Q-\sum_{n=1}^{N} w_{n} P_{0}(t)
$$

for $n=0$, and

$$
\frac{d P_{n}(t)}{d t}=w_{n} P_{0}(t)-k P_{n}(t)
$$

for $n>0$. The physical meaning of these equations is that morphogens are produced at the origin cell and then they are transported to the target cells. There is a distribution of arrival times for 
the signaling molecules to reach each target cell. The average arrival time is equal to $1 / w_{n}$. One should note also here that generally the morphogens come to different cells at different times.

Assuming that initially there were no morphogens in the system, $P_{n}(t=0)=0$ for all $n$, these master equations can be solved exactly at all times, yielding

$$
\begin{gathered}
P_{0}(t)=\frac{Q}{\eta}\left[1-e^{-\eta t}\right] ; \\
P_{n}(t)=\left[\frac{Q w_{n}}{\eta(\eta-k)}\right] e^{-\eta t}-\left[\frac{Q w_{n}}{k(\eta-k)}\right] e^{-k t}+\frac{Q w_{n}}{\eta k}
\end{gathered}
$$

where $\eta=\sum_{n=1}^{N} w_{n}$ is defined as a total transportation rate along all cytonemes. One can see that the concentration of signaling molecules at each cell varies exponentially with time, and it is a result of balancing two opposing processes: the direct delivery with the rate $\eta$ and the removal with the rate $k$. For the special case of $k=\eta$ we obtain $P_{n}(t)=\frac{Q w_{n}}{k^{2}}\left[1-e^{-k t}\right]$. At large times $(t \rightarrow \infty)$, the density profiles reach stationary values,

$$
P_{n}^{(s)}=\frac{Q}{k \eta} w_{n}, \quad P_{0}^{(s)}=\frac{Q}{\eta}
$$

To understand the mechanisms of the direct delivery of signaling molecules, it is important to consider the approach to the stationary-state behavior. It can be quantified by calculating a local relaxation function $R_{n}(t) \equiv \frac{P_{n}(t)-P_{n}^{(s)}}{P_{n}(0)-P_{n}^{(s)}}$. ${ }^{14}$ This function can be viewed as a relative measure of how close is the system to the stationary state at the given location. Our calculations produce

$$
R_{n}(t)=\frac{\eta e^{-k t}-k e^{-\eta t}}{\eta-k}, \quad R_{0}(t)=e^{-\eta t}
$$

The local relaxation function can be used for evaluating a local accumulation time (LAT), $t_{n}=$ $\int_{0}^{\infty} R_{n}(t) d t,{ }^{14}$ which is defined as a time when the stationary concentration at the given location 
can be achieved for the first time. It can be easily shown that

$$
<t_{n}>=\frac{1}{k}+\frac{1}{\eta}, \quad<t_{0}>=\frac{1}{\eta} .
$$

Since there is no dependence on the target cell index $n$ these calculations lead to an important conclusion that the relaxation dynamics to the stationary behavior is identical at all target cells. This is a surprising result, which also sharply contrasts with the position-dependent approach to the stationary phase in the indirect diffusional delivery of morphogens. ${ }^{14,16,19}$ This can be understood using the following arguments. In the delivery of signaling molecules via cytonemes the behavior at each target cell is independent of other cells. In this case, the relaxation dynamics is governed by two processes: achieving the stationary state at the source cell $(n=0)$ and the degradation of morphogens at the target cells $(n \geq 1)$, which is taking place with the same rate $k$. For the free extracellular diffusion reaching by signaling molecules the specific locations for the first time is the most critical step. ${ }^{16}$ Obviously, these first arrival processes are position dependent since the diffusional front moves sequentially from the source cell to the targets cells.

Analyzing the formation of biological signaling profiles, it is important to compare the robustness of direct and indirect delivery mechanisms. There are many ways to describe the robustness. ${ }^{8}$ However, there is no agreed framework for analyzing robustness and thus many different ways of evaluating this important property can be utilized. Here we use the normalized variance as a quantitative measure of fluctuations and noise in the delivering of morphogen molecules to specific target cells. This approach explicitly evaluates the ability of the system to reach the specified stationary-state concentrations at specific time intervals. From this point of view, it directly reflects the stochasticity of the underlying reaction-diffusion processes in the formation of morphogen gradients. One expects that more robust systems show less fluctuations and noise. To perform such calculations, we need the second moment of LAT, which can be also obtained from the local relax- 
ation function, ${ }^{14,19}<t_{n}^{2}>=-\int_{0}^{\infty} t^{2} \frac{d R_{n}(t)}{d t} d t$, which leads to

$$
<t_{n}^{2}>=\frac{2\left(\eta^{2}+k \eta+k^{2}\right)}{k^{2} \eta^{2}}, \quad<t_{0}^{2}>=\frac{2}{\eta^{2}}
$$

The normalized variance is defined then as,

$$
\sigma_{n}=\left[\frac{<t_{n}^{2}>-<t_{n}>^{2}}{<t_{n}>^{2}}\right]^{1 / 2}
$$

which yields for the direct delivery mechanism,

$$
\sigma_{n}=\left[\frac{\eta^{2}+k^{2}}{\eta^{2}+2 k \eta+k^{2}}\right]^{1 / 2}, \quad \sigma_{0}=1
$$

From this expression we find that the normalized variance is always less or equal to one for any target cell, and it reaches the minimum when $\eta=k$, giving $\sigma_{n}=1 / \sqrt{2}$. The largest variance, $\sigma_{n} \simeq 1$, is achieved when one of the relevant rates dominates, i.e., for $\eta \gg k$ or $k \gg \eta$.

The normalized variances for the translocation of morphogens through cytonemes and via the free extracellular diffusion are presented in Fig. 2. One can see that there is always less fluctuations and noise in the direct delivery mechanism, and the largest difference is achieved when the degradation and the total transportation rates are comparable. This suggests that moving the morphogens across cytonemes provides a more robust mechanism of delivery biological signals. It can be explained by noticing that the passive diffusion mechanism is more stochastic because molecules can fluctuate spatially between different cells. In the direct delivery mechanism such option does not exist. However, it should be mentioned here that this conclusion also depends on the assumption of our model that cytoneme connections are stable during the formation of signaling profiles.

Our theoretical analysis indicates the importance of the transportation rates in the direct delivery of morphogens. To understand the mechanisms of the transport through cytonemes, a more microscopic description of $w_{n}$ is needed. There are no quantitative measurements of these rates, ${ }^{6}$ 

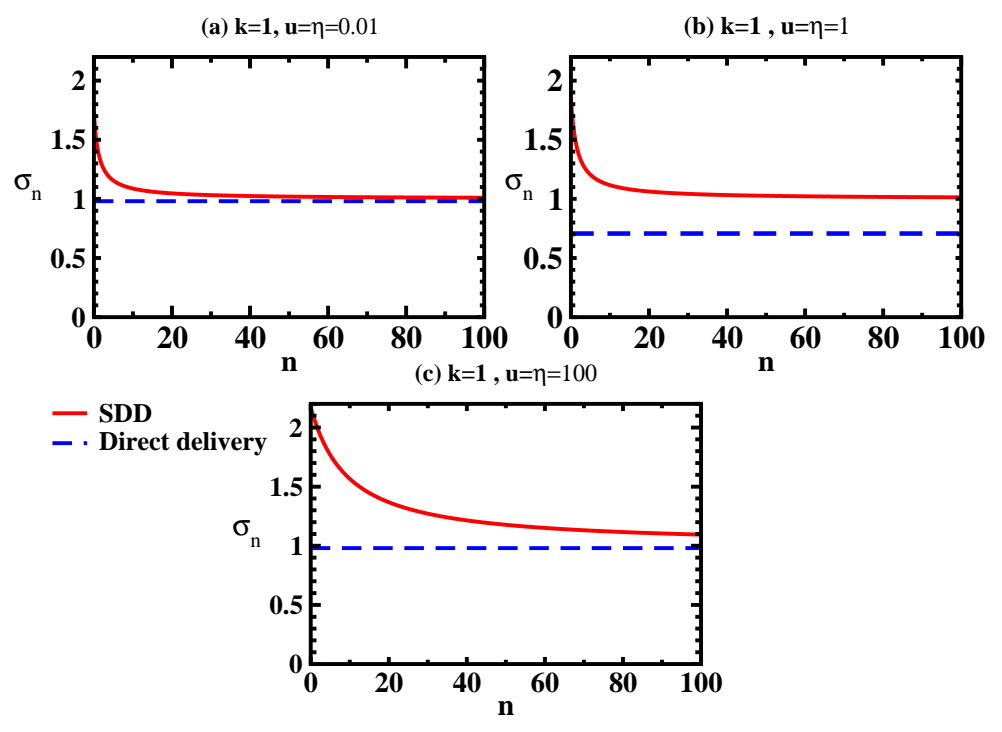

Figure 2: Normalized variance as a function of the distance from the source cell for direct and indirect delivery mechanisms. Solid lines correspond to the SDD model with a diffusion rate $u .{ }^{16}$ Dashed lines correspond to the translocation of morphogens via cytonemes with the total transportation rate $\eta$. 
but based on observations that the motion of motor proteins along the cytsokeleton might be involved, ${ }^{26}$ we propose the following two limiting models. Our first hypothesis is that the rate of moving along the cytoneme is given by a free-energy difference between finding the morphogen at the cell $n$ and at the source cell,

$$
w_{n}=w_{n}^{(0)} \exp \left[-\frac{\Delta G(n)}{k_{B} T}\right]
$$

where $\Delta G(n)$ is the energy required to displace the morphogen from the source to the target cell $n$, and $w_{n}^{(0)}$ is a rate for a case when $\Delta G(n)=0$. Let us assume here that the length of the cytoneme that connects the source and the cell is, $L_{n}$ is proportional to $n$, i.e., $L_{n}=A n$. Furthermore, we assume that the motor proteins spend energy $\varepsilon$ (in units of $k_{B} T$ ) by moving every signaling molecule a distance $l$. The free energy difference can be written as

$$
\Delta G(n)=\frac{L_{n} \varepsilon k_{B} T}{l}=\frac{A n \varepsilon k_{B} T}{l}=\frac{n k_{B} T}{a},
$$

where $a=l / A \varepsilon$. Then, the explicit expression for the transportation rate is given by $w_{n}=w_{n}^{(0)} \exp \left[-\frac{n}{a}\right]$. This leads to the following stationary density profile of signaling molecules

$$
P_{n}^{(s)}=\frac{Q}{k \eta} w_{n}^{(0)} \exp \left[-\frac{n}{a}\right]
$$

where the total transportation rate $\eta$ is equal to

$$
\eta=\sum_{n=1}^{N} w_{n}^{(0)} \exp \left[-\frac{n}{a}\right]=w_{n}^{(0)} \frac{\left(\exp \left[-\frac{1}{a}\right]-\exp \left[-\frac{(1+N)}{a}\right]\right)}{1-\exp \left[-\frac{1}{a}\right]}
$$

This model predicts the exponential decaying stationary-state density profile [see Eq. (13)], and the decay length, specified by the parameter $a$, is larger for more efficient motor proteins that spend less energy in driving the morphogens along the cytonemes. The exponential morphogen gradients are also found for the indirect free diffusion delivery of the signaling molecules at large 
times. $5,6,16$ However, in this case the decay length is determined by the ratio of diffusion and degradation rates. ${ }^{14,16}$ This underlies the importance of energy dissipation in the transportation of signaling molecules through cytonemes.

In the direct delivery route, the morphogens are moved along effectively one-dimensional structures, ${ }^{6,26}$ and this suggest that intermolecular interactions might strongly influence the translocation dynamics. For example, morphogens cannot pass each other due to exclusion interactions. From this point of view, our first model describes the transportation of morphogens that do not interact with each other inside of the cytonemes. In real systems, one expects that signaling molecules might interact with each other during the translocation. This is the basis for our second model of the transportation. Here we note that the motion of signaling molecules in each cytoneme can be viewed as 1D multi-particle biased transport. This can be well described by totally asymmetric simple exclusion processes (TASEP), which were successfully employed for analyzing many complex nonequilibrium phenomena in chemistry, physics and biology. ${ }^{32}$ We assume that each cytoneme can be viewed as a lattice segment with $n$ sites: see Fig. 1. Signaling molecules are hoping only in the direction of the target cell from one site to the next one. To each lattice site $i$ $(1 \leq i \leq n)$ we assign an occupation number $\tau_{i}$ which is zero if the site is empty or $\tau_{i}=1$ if the site is occupied. This means that only one molecule can be found at each site, and morphogens interact with each other via hard-core exclusions. The particle at site $i$ can jump forward to the site $i+1$ with the rate 1 , provided that this site is empty. The particle can enter the lattice with a rate $\alpha$ from the left boundary if the first site is empty, and it can also leave the lattice segment (cytoneme) from the last site with a rate $\beta$. The steady-state properties for the TASEP on finite lattice segments with open boundaries have been calculated exactly. ${ }^{31,32}$ The particle flux through the segment of length $n$ is given by, 31

$$
J(\alpha, \beta ; n)=\frac{S_{n-1}(1 / \beta)-S_{n-1}(1 / \alpha)}{S_{n}(1 / \beta)-S_{n}(1 / \alpha)},
$$

where an auxiliary function $S_{n}(y)$ is defined as,

$$
S_{n}(y)=\sum_{i=0}^{n-1} \frac{(n-i)(n+i-1) !}{n ! i !} y^{n-i+1} .
$$


It is also possible to evaluate explicitly the occupation of every lattice site in all segments. ${ }^{31}$ One should also discuss the units of the flux $J(\alpha, \beta ; n)$ in Eq. (15). Because the rates $\alpha$ and $\beta$ are expressed in (time) $)^{-1}$, the function $S_{n}(y)$ (with $y=1 / \alpha$ or $y=1 / \beta$ ) has a dimension of (time $)^{n+1}$ [see Eq. (16) and Ref. ${ }^{31}$ ]. As a result, the units of the flux $J(\alpha, \beta ; n)$ are inverse time, as expected.

To connect TASEP with the transport through cytonemes with $N$ target cells we can identify the effective entrance rate as $\alpha=Q / N$ since $Q$ is the total rate of production of signaling molecules in the source cell and we assume that they are split equally between all cytonemes going to $N$ target cells. The exit rate can be associated with the degradation rate $k$ that removes the particles from the cytonemes, i.e., $\beta=k$. It is also reasonable to connect the transportation rate $w_{n}$ with the particle flux through the cytoneme to the $n$-th target cell, $w_{n}=J\left(\frac{Q}{N}, k ; n\right)$. At stationary-state conditions it leads us to explicit expression for the density profile,

$$
P_{n}^{(s)}=\frac{Q}{k \eta} J\left(\frac{Q}{N}, k ; n\right)=\frac{Q}{k \eta}\left[\frac{S_{n-1}(1 / k)-S_{n-1}(N / Q)}{S_{n}(1 / k)-S_{n}(N / Q)}\right],
$$

where the total transportation rate is given by

$$
\eta=\sum_{n=1}^{N}\left[\frac{S_{n-1}(1 / k)-S_{n-1}(N / Q)}{S_{n}(1 / k)-S_{n}(N / Q)}\right]
$$

The stationary-state density profiles obtained for the direct delivery mechanism for interacting and non-interacting morphogens are presented in Fig. 3. One can see that for target cells that are close to the source the behavior is similar in both cases, while for the cells located much further away there is a saturation behavior due to exclusion interactions between morphogens. Because the profiles are expected to be very non-uniform in order to efficiently transfer the information, this suggests that the direct delivery of interacting morphogens might not be the most efficient mechanism of transporting the biological signals on very long distances if exclusion interactions are important. However, it is important to mention that the presented model is rather oversimplified and some important biological phenomena are be included here. The dynamics of the signaling transport might strongly deviate from these predictions. For example, the presence of interme- 
diate states during the transport of morphogens across the cytonemes might also add significant fluctuations to the system, and this might influence our predictions about the robustness of the system.

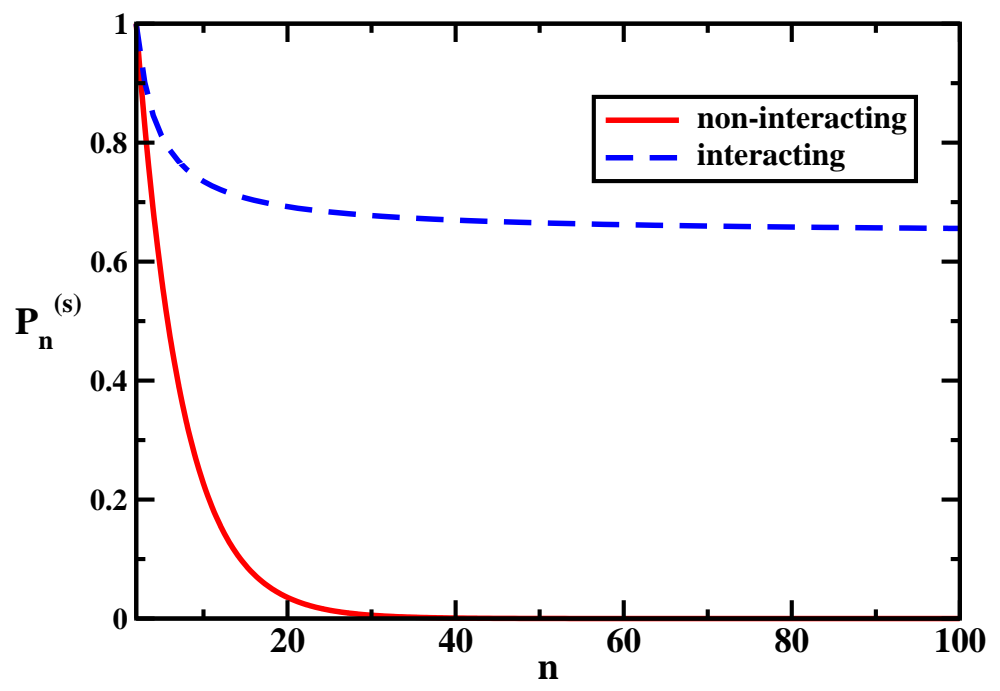

Figure 3: Stationary density profiles for interacting and non-interacting morphogens in the direct delivery mechanism. The parameters used for calculations are: $k=0.9, \eta=5.6, N=100$ and $a=2.45$

In this work it was assumed that morphogens start to work only after reaching their stationarystate profiles. However, there are indications that in many systems the morphogenetic signals are decoded already for the transient conditions before coming to the steady state. ${ }^{34}$ Our analysis can be extended for estimating the times to reach any concentrations of signaling molecules at the target cells. It will be interesting to compare the pre-steady-state coding mechanisms for direct and indirect delivery mechanisms.

To summarize, we developed a comprehensive theoretical framework for analyzing a new mechanism of transferring biological signals that utilizes direct delivery of morphogens via the cellular extensions cytonemes. Recent experimental studies indicate that this mechanism might be 
important in the development of multi-cellular living systems. Our calculations predict that the transport through cytonemes simultaneously transfers the biological information to all target embryo cells. The critical role in the direct delivery dynamics is played by the translocation rates along the cytonemes and degradation rates. It is shown that the transportation via cytonemes is more robust than the free extracellular diffusion for delivering the biological information. However, it requires energy dissipation for effective functioning. In addition, we found that intermolecular interactions between morphogens inside the cytonemes might limit the efficiency of this mechanism to only target cells that are close to the source region. Although our theoretical analysis provides a fully quantitative description of the direct delivery mechanisms of signaling molecules and it explains its main characteristics, it should be noted that the presented method is still oversimplified with many important features not considered. For example, we assume that the cytonemes are static structures, while the experimental studies clearly indicate that the cytonemes might extend

and retract dynamically. ${ }^{30}$ It will be important to test the proposed theoretical ideas in experimental studies as well as in more advanced theoretical investigations.

\section{Acknowledgments}

The work was supported by the Welch Foundation (Grant C-1559), by the NSF (Grant CHE1360979), and by the Center for Theoretical Biological Physics sponsored by the NSF (Grant PHY-1427654). We also would like to thank Tom Kornberg, Stas Shvartsman and Ruth Baker for useful comments and suggestions.

\section{References}

(1) Martinez-Arias, A.; Stewart, A. Molecular Principles of Animal Development; Oxford University Press: New York, 2002.

(2) Wolpert, L. Principles of Development. Oxford University Press: New York, 1998. 
(3) Kerszberg, M.; Wolpert, L. Specifying Positional Information in the Embryo: Looking Beyond Morphogens. Cell 2007, 130, 205.

(4) Lander, A.D. Morpheus Unbound: Re-imagining the Morphogen Gradient. Cell 2007, 128, 245-256.

(5) Porcher, A.; Dostatni, N. The Bicoid Morphogen System. Curr. Biol. 2010, 20, R249-R254.

(6) Kornberg, T. B. The Imperatives of Context and Contour for Morphogen Dispersion. Biophys. J. 2012, 103, 2252-2256.

(7) Wartlick, O.; Kicheva, A.; Gonzales-Gaitan, M. Morphogen Gradient Formation Cold Spring Harb. Perspect. Biol. 2009, 1, a001255.

(8) Gregor, T.; Wieschaus, E.F.; McGregor, A.P.; Bialek W.; Tank, D.W.; Stability and Nuclear Dynamics of the Bicoid Morphogen Gradient Cell 2007, 130, 141-152.

(9) Kicheva, A.; Pantazis, P.; Bollenbach, T.; Kalaidzidis, Y.; Bittig, T.; Jülicher, F.; GonzalesGaitan, M. Kinetics of Morphogen Gradient Formation. Science 2007, 315, 521-525.

(10) Yu, S.R.; Burkhardt, M.; Nowak, M.; Ries, J.; Petrasek, Z.; Scholpp, S.; Schwille, P.; Brand, M. Fgf8 Morphogen Gradient Forms by a Source-Sink Mechanism with Freely Diffusing Molecules. Nature 2009, 461, 533-536.

(11) Drocco, J.A.; Grimm, O.; Tank, D.W.; Wieschaus, E. Measurement and Perturbation of Morphogen Lifetime: Effects on Gradient Shape. Biophys. J. 2011, 101, 1807-1815.

(12) Spirov, A.; Fahmy, K.; Schneider, M.; Frei, E.; Noll, M.; Baumgartner, S. Formation of the Bicoid Morphogen Gradient: an mRNA Gradient Dictates the Protein Gradient. Development 2009, 136, 605-614.

(13) Little, S.C.; Tkacik, G.; Kneeland, T.B.; Wieschaus, E.; Gregor, T. The Formation of the Bicoid Morphogen Gradient Requires Protein Movement from Anteriorly Localized mRNA. PLoS Biol. 2011, 9, e1000596. 
(14) Berezhkovskii, A. M.; Sample, C.; Shvartsman, S. Y. How Long Does It Take to Establish a Morphogen Gradient? Biophys. J. 2010, 99, L59-L61.

(15) Fedotov, S.; Falconer, S. Nonlinear Degradation-Enhanced Transport of Morphogens Performing Subdiffusion. Phys. Rev. E 2014, 89, 012107-6.

(16) Kolomeisky, A. B. Formation of a Morphogen Gradient: Acceleration by Degradation. J. Phys. Chem. Lett. 2011, 2, 1502-1505.

(17) Gordon, P. V.; Muratov, C. B.; Shvartsman, S. Y. Local Accumulation Times for Source, Diffusion, and Degradation Models in Two and Three Dimensions. J. Chem. Phys. 2013, $138,104121$.

(18) England, J.L.; Cardy, J. Morphogen Gradient from a Noisy Source. Phys. Rev. Lett. 2005, 94, 078101-4.

(19) Teimouri, T.; Kolomeisky, A. B. Development of Morphogen Gradient: The Role of Dimension and Discreteness. J. Chem. Phys. 2014, 140, 085102-14.

(20) Verbeni, M.; Sanchez, O., Mollica, E.; Siegl-Cachedenier, I.; Carleton, A.; Guerrero, I., Ruiz i Altaba, A.; Soler, J. Morphogenetic Action through Flux-Limited Spreading. Phys. Life Rev. 2013, $10,457-475$.

(21) Kornberg, T. B.; Roy, S. Communicating by Touch-Neurons Are not Alone. Trends Cell. Biol. 2014, $24,370-376$.

(22) Roy, S.; Kornberg, T. B. Paracrine Signaling Mediated at Cell-Cell Contacts. Bioessays 2014, $37,25-33$.

(23) Fairchild, C. L.; Barna, M. Specialized Filopodia: at the 'Tip' of Morphogen Transport and Vertebrate Tissue Patterning. Current Opinion in Genetics and Development 2014, 27, 67-73.

(24) Rørth, P. Reach Out and Touch Someone. Science 2014, 343, 848-849. 
(25) Kornberg, T. B.; Roy, S. Cytonemes as Specialized Signaling Filopodia. Development 2014, $141,729-736$.

(26) Sanders, T. A.; Llagostera, E; Barna, M. Specialized Filopodia Direct Long-Range Transport of SHH During Vertebrate Tissue Patterning. Nature 2013, 479, 628-632.

(27) Gradilla, A-C; Guerrero, I. Cytoneme-Mediated Cell-to-Cell Signaling During Development. Cell. Tissue. Res. 2013, 352, 59-66.

(28) Guerrero, I.; Kornberg, T. B. Specialized Filopodia Direct Long-Range Transport of SHH During Vertebrate Tissue Patterning. Seminars in Cell and Developmental Biology 2014, 33, $52-62$.

(29) Müller, P.; Rogers, K. W.; Yu, S. R.; Brand M.; Schier, A. F. Morphogen Transport. Development 2013, 140, 1621-1638.

(30) Bischoff, M.; Gradilla, A-C.; Seijo, I.; Andrés, G.; Rodríguez-Navas, C.; González-Méndez, L.; Guerrero, I. Cytonemes Are Required for the Establishment of a Normal Hedgehog Morphogen Gradient in Drosophila Epithelia. Nature Cell Biology 2013, 15, 1269-1281.

(31) Derrida, B.; Evans, M.R.; Hakim, V.; Pasquier, V.; Exact Solution of a 1D Asymmetric Exclusion Model Using a Matrix Formulation. J. Phys. A: Gen. Math. 1993, 26, 1493-1517.

(32) Chou, T.; Mallick K.; Zia, R. K. P. Non-Equilibrium Statistical Mechanics: From a Paradigmatic Model to Biological Transport. Rep. Prog. Phys 2011, 74, 116601(1-41).

(33) Cohen, M.; Georgiou, M.; Stevenson, N. L.; Miodownik, M.; Buzz, B. Dynamic Filopodia Transmit Intermittent Delta-Notch Signaling to Drive Pattern Refinement During Lateral Inhibition. Developmental Cell 2010, 19, 78-89.

(34) Richards, D. M.; Saunders, T. E. Spatiotemporal Analysis of Different Mechanisms for Interpreting Morphogen Gradients. Biophys. J. 2015, 108, 2061-2073. 